\title{
AFFINE INVARIANT CONDITIONS \\ FOR THE TOPOLOGICAL DISTINCTION \\ OF QUADRATIC SYSTEMS \\ WITH A CRITICAL POINT OF THE 4TH MULTIPLICITY
}

\author{
Mark Voldman, Iu. T. Calin and N. I. Vulpe
}

Abstract

The affine invariant partition of the set of quadratic systems with one finite singular point of the 4th multiplicity with respect to different topological classes is accomplished. The conditions corresponding to this partition are semi-algebraic, i.e. they are expressed as equalities or inequalities between polynomials.

Let us consider the system of differential equations

$$
\frac{d x^{j}}{d t}=a^{j}+a_{\alpha}^{j} x^{\alpha}+a_{\alpha \beta}^{j} x^{\alpha} x^{\beta}, \quad(j, \alpha, \beta=1,2)
$$

where $a_{\alpha}^{j}$ and $a_{\alpha \beta}^{j}(j, \alpha, \beta=1,2)$ are real numbers (the tensor $a_{\alpha \beta}^{j}$ is symmetric in the lower indices, with respect to which the complete contraction was made).

The topological classification of the quadratic system (1) in the case when it has a unique real critical point on its phase plane is done in [4] and [6]. The conditions for the classification into topological classes described in these papers are expressed using the parameters of the corresponding canonical forms. The topological classification of system (1) with a unique real simple critical point is done in [7].

In $[\mathbf{9}]$ the conditions for the topological classification of quadratic systems with a unique finite critical point of multiplicity 4 were gived. However, the critical point was situated at the origin of the coordinates of system (1) and the obtained conditions are center affine invariant. Thus 
the conditions given in [9] are not valid for the full system (1) in the case when such a point does not coincide with the origin.

Using the results of article [9] we shall find the corresponding affine invariant partition of coefficient space $E^{12}$ of system (1). The affine invariant conditions corresponding to this partition are semi-algebraic, i.e. they are expressed as equalities or inequalities between polynomials.

\section{Preliminaires}

Let $a \in E^{12}$ be an element of the space of the coefficients of system (1) and let us consider the group $Q$ of nondegenerate real linear transformations of the phase plane. We denote by $r_{q}$ the linear presentation of any element $q \in Q$ into the coefficient space $E^{12}$ of system (1).

Definition 1 [8]. A polynomial $K(a, x)$ of the coefficients of system (1) and the unknown variables $x^{1}$ and $x^{2}$ is called a comitant of system (1) in the group $Q$, if there exists a function $\lambda(q)$ such that

$$
K\left(r_{q} \cdot a, q \cdot x\right) \equiv \lambda(q) K(a, x)
$$

for every $q \in Q, a \in E^{12}$ and $x=\left(x^{1}, x^{2}\right)$.

The function $\lambda(q)$ is called a multiplicator. If $\lambda(q) \equiv 1$, then the comitant $K(a, x)$ is called absolute; otherwise it is called relative. It is known (see $[8]$ ), that $\lambda(q)=\Delta_{q}^{-x}$, where $\Delta_{q} \neq 0$ is the determinant of the linear transformation matrix and the integer $\varkappa$ is called the weight of the comitant. A comitant $K$ of system (1) in the group $Q=G L(2, R)$ of linear homogeneous transformations of the phase plane of system (1) (which is also called a group of center-affine transformations) is called center affine. A comitant $K$ of system (1) in the group $Q=A f f(2, R)$ of affine (linear non-homogenous) transformations is called affine. If the comitant $K$ does not depend explicitly on the variables $x^{1}$ and $x^{2}$ then it is called an invariant (center affine or affine, respectively).

Remark 1. We say that the comitant of system (1) equals zero when all its coefficients vanish. The signs of the comitants which take part in some sequences of conditions should be calculated at one and the same point, where they do not vanish.

We denote by $T(2, R)$ the group of shift transformations and by $r_{t}$ the linear presentation of any element $t \in T$ into the coefficient space $R^{12}$ of system (1). 
Definition 2 [3]. A center affine comitant $K(a, x)$ of system (1) is called a $T$-comitant if the relation

$$
K\left(r_{t} \cdot a, x\right) \equiv K(a, x)
$$

is valid for every $t \in T$ and $a \in E^{12}$.

We shall say that comitant $K$ is of the type $(\rho, \varkappa, d)$ if it is a homogeneous polynomial of degrees $\rho$ and $d$ in the coordinates of vector $x$ and in the coefficients of system (1), respectively, and if its weight is equal to $\varkappa$.

Definition 3 [5]. The polynomial

$$
(f, \varphi)^{(k)}=\frac{(r-k) !(\rho-k) !}{r ! \rho !} \sum_{h=0}^{k}(-1)^{h} C_{k}^{h} \frac{\partial^{k} f}{\partial\left(x^{1}\right)^{k-h} \partial\left(x^{2}\right)^{h}} \frac{\partial^{k} \varphi}{\partial\left(x^{1}\right)^{k} \partial\left(x^{2}\right)^{k-h}}
$$

is called a transvectant of index $k$ of two forms $f$ and $\varphi$. The degree of these forms in the coordinates of vector $x=\left(x^{1}, x^{2}\right)$ are equal to $r$ and $\rho$, respectively and $k \leq \min (r, \rho)$.

Proposition 1 [3]. The transvectant $(f, \varphi)^{(k)}$ of two T-comitants $f$ and $\varphi$ of the types $\left(r, \varkappa_{1}, d_{1}\right)$ and $\left(\rho, \varkappa_{2}, d_{2}\right)$ respectively will be also a $T$-comitant of the type $\left(r+\rho-2 k, \varkappa_{1}+\varkappa_{2}+k, d_{1}+d_{2}\right)$.

Let us write system (1) as

$$
\begin{aligned}
& \frac{d x^{1}}{d t}=P_{0}+P_{1}+P_{2}, \\
& \frac{d x^{2}}{d t}=Q_{0}+Q_{1}+Q_{2},
\end{aligned}
$$

where $P_{i}(i=0,1,2)$ are homogeneous polynomials of degree $i$, and consider the following center affine invariants and comitants, which are 
constructed directly through the right-side parts of the given system:

(2)

$$
\begin{aligned}
J_{1} & =\left|\begin{array}{ll}
\partial P_{1} / \partial x^{1} & \partial P_{1} / \partial x^{2} \\
\partial Q_{1} / \partial x^{1} & \partial Q_{1} / \partial x^{2}
\end{array}\right|=a_{p}^{\alpha} a_{q}^{\beta} \varepsilon_{\alpha \beta} \varepsilon^{p q}, \\
C_{1} & =\left|\begin{array}{ll}
\partial P_{1} / \partial x^{1} & \partial P_{2} / \partial x^{2} \\
\partial Q_{1} / \partial x^{1} & \partial Q_{2} / \partial x^{2}
\end{array}\right|-\left|\begin{array}{cc}
\partial P_{1} / \partial x^{2} & \partial P_{2} / \partial x^{1} \\
\partial Q_{1} / \partial x^{2} & \partial Q_{2} / \partial x^{1}
\end{array}\right| \\
& =x^{\alpha} a_{q}^{\beta} a_{p \alpha}^{\gamma} \varepsilon_{\beta \gamma} \varepsilon^{p q}, \\
C_{2} & =\left|\begin{array}{ll}
P_{0} & P_{1} \\
Q_{0} & Q_{1}
\end{array}\right|=x^{\alpha} a^{\beta} a_{\alpha}^{\gamma} \varepsilon_{\beta \gamma}, \\
C_{3} & =\frac{1}{4}\left|\begin{array}{ll}
\partial P_{2} / \partial x^{1} & \partial P_{2} / \partial x^{2} \\
\partial Q_{2} / \partial x^{1} & \partial Q_{2} / \partial x^{2}
\end{array}\right|=\frac{1}{2} x^{\alpha} x^{\beta} a_{p \alpha}^{\gamma} a_{q \beta}^{\delta} \varepsilon_{\gamma \delta} \varepsilon^{p q}, \\
C_{4} & =\left|\begin{array}{ll}
P_{0} & P_{2} \\
Q_{0} & Q_{2}
\end{array}\right|=x^{\alpha} x^{\beta} a^{\gamma} a_{\alpha \beta}^{\delta} \varepsilon_{\gamma \delta}, \\
C_{5} & =\left|\begin{array}{ll}
P_{1} & P_{2} \\
Q_{1} & Q_{2}
\end{array}\right|=x^{\alpha} x^{\beta} x^{\gamma} a_{\alpha}^{\delta} a_{\beta \gamma}^{\mu} \varepsilon_{\delta \mu}, \\
C_{6} & =x^{2} P_{2}-x^{1} Q_{2}=x^{\alpha} x^{\beta} x^{\gamma} a_{\alpha \beta}^{\delta} \varepsilon_{\delta \gamma},
\end{aligned}
$$

(where $\varepsilon^{11}=\varepsilon^{22}=\varepsilon_{11}=\varepsilon_{22}=0, \varepsilon^{12}=\varepsilon_{12}=-\varepsilon^{21}=-\varepsilon_{21}=1$ ).

We also introduce the following $T$-comitants:

$$
\begin{aligned}
& C_{7}=x^{q} x^{s} x^{v}\left[2 a^{p} a_{\alpha \gamma}^{r}-a_{\alpha}^{p} a_{\gamma}^{r}\right] a_{\beta \varkappa}^{u} \varepsilon_{p q} \varepsilon_{r s} \varepsilon_{u v} \varepsilon^{\alpha \beta} \varepsilon^{\gamma \varkappa}, \\
& C_{8}=x^{\alpha} x^{\beta}\left[a_{s}^{m} a_{\beta}^{n} a_{p r}^{k}-2 a_{r}^{k} a_{\beta}^{n} a_{p s}^{m}+a_{p}^{k} a_{r}^{m} a_{s \beta}^{n}-4 a^{m} a_{p r}^{k} a_{s \beta}^{n}\right] a_{q \alpha}^{l} \varepsilon_{k l} \varepsilon_{m n} \varepsilon^{p q} \varepsilon^{r s},
\end{aligned}
$$

and transvectants:

$$
\begin{aligned}
\mu_{1} & =\left(C_{3}, C_{3}\right)^{(2)}, H_{1}=\left(C_{3}, C_{1}\right)^{(1)}, \\
G_{1} & =\left(C_{1}, C_{5}\right)^{(1)}, G_{2}=\left(C_{5}, C_{5}\right)^{(2)}, G_{3}=\left(C_{3}, C_{4}\right)^{(1)}, \\
\eta_{1} & =\left(\left(\left(C_{6}, C_{6}\right)^{(2)}, C_{6}\right)^{(1)}, C_{6}\right)^{(3)}, M_{1}=\left(C_{6}, C_{6}\right)^{(2)}, \\
\bar{D}_{1} & =\left(\left(\left(C_{6}, C_{7}\right)^{(2)}, C_{6}\right)^{(1)}, C_{6}\right)^{(3)}, \bar{D}_{2}=\left(\left(C_{3}, C_{7}\right)^{(1)}, C_{6}\right)^{(3)}, \\
\bar{D}_{3} & =\left(C_{3}, C_{8}\right)^{(2)}, \bar{D}_{4}=\left(\left(\left(C_{7}, C_{7}\right)^{(2)}, C_{7}\right)^{(1)}, C_{7}\right)^{(3)},
\end{aligned}
$$

which by Proposition 1 are also $T$-comitants. 
We introduce the following notation

(3)

$$
\begin{aligned}
\mu & =-2 \mu_{1}, H=2 H_{1}, 2 G=4 G_{1}-3 G_{2}+8 G_{3}, \\
F & =J_{1} C_{5}+2 C_{1} C_{4}+4 C_{2} C_{3}, V=C_{4}^{2}-C_{2} C_{5}, \\
2 \eta & =27 \eta_{1}, 2 M=9 M_{1}, L=C_{6}, D=-\bar{D}_{4}, \\
D_{1} & =\bar{D}_{1}-2 \bar{D}_{2}, D_{2}=4 \bar{D}_{2}+\bar{D}_{3}, D_{3}=\bar{D}_{1}\left(21 \bar{D}_{1}-26 \bar{D}_{2}+4 \bar{D}_{3}\right) .
\end{aligned}
$$

As it was shown in [2] the comitants $\mu, H, G, F$ and $V$ are responsible for the number and multiplicities of the finite singular points of quadratic system (1).

Definition 4. We shall say that the real finite singular point $M_{0}$ of quadratic system $a \in E^{12}$ has multiplicity $k$ (or, in other words, from the singular point $M_{0}$ bifurcate $k$ singular points, as the coefficients of system $a \in E^{12}$ are varied), if the following conditions are satisfied:

(i) there exist a positive $\varepsilon_{0}>0$ and $\delta_{0}>0$ such that in the neighbourhood $U\left(a, \delta_{0}\right)$ of the point $a$, there are no points, which correspond to a system (1) having more than $k$ singular points in the neighbourhood $U\left(M_{0}, \varepsilon_{0}\right)$ of the singular point $M_{0}$;

(ii) for every positive $\delta<\delta_{0}$ and $\varepsilon<\varepsilon_{0}$, there is a point $b \in$ $U(a, \delta)$ which corresponds to a system (1) with $k$ singular points in $U\left(M_{0}, \varepsilon\right)$.

According to $[\mathbf{2}]$ we shall construct the following $T$-comitants:

$$
\begin{aligned}
& P=G^{2}-6 F H+12 \mu V, R=4\left(3 H^{2}-2 \mu G\right), \\
& T=2 \mu\left[2 G^{3}+9 \mu\left(3 F^{2}-8 G V\right)-18 F G H+108 H^{2} V\right]-P R .
\end{aligned}
$$

Proposition 2 [2]. Quadratic system (1) has a finite singular point of multiplicity 4 if, and only if, the following conditions hold:

$$
\mu \neq 0, D=0, P=R=T=0 .
$$




\section{Main result}

Theorem. A phase portrait of quadratic system (1) with a finite point of multiplicity $4(\mu \neq 0, D=P=R=T=0)$ in the Poincare disk is up to a homeomorphism given by:

Figure 1 if $\eta>0, D_{1}<0, D_{2}>0$;

Figure 2 if $\eta>0, D_{1}<0, D_{2}<0$ or $\eta>0, D_{1} \geq 0$;

Figure 3 if $\eta=0, M \neq 0, D_{1}<0, D_{2}>0, D_{3} \geq 0$;

Figure 4 if $\eta=0, M \neq 0, D_{1}<0, D_{2}>0, D_{3}<0$;

Figure 5 if $\eta=0, M \neq 0, D_{1}<0, D_{2}<0$ or $\eta=0, M \neq 0, D_{1} \geq 0$;

Figure 6 if $\eta<0$, or $\eta=M=0, L \neq 0$.

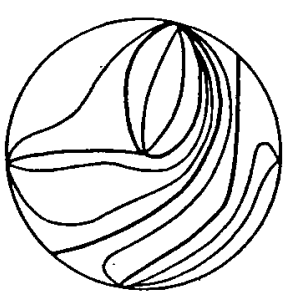

Figure 1

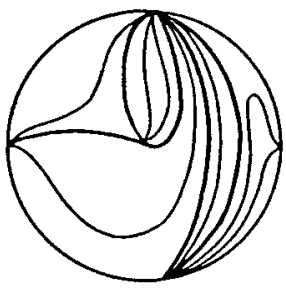

Figure 4

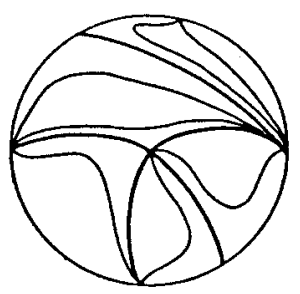

Figure 2

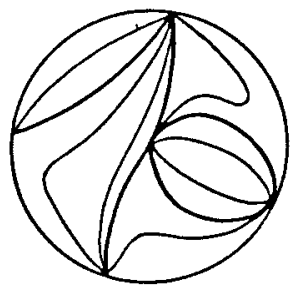

Figure 5

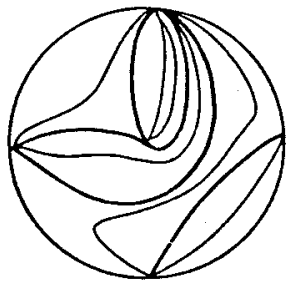

Figure 3

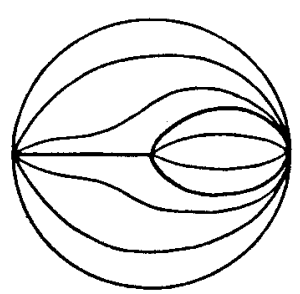

Figure 6

Proof: Let us assume that conditions (5) are satisfied, i.e. according to (6) there is a singular point of multiplicity 4 arbitrarily situated on the phase plane of system (1). By applying a shift transformation we can move the origin of the coordinates to this point. Thus we obtain the system

$$
\frac{d x^{j}}{d t}=a_{\alpha}^{j} x^{\alpha}+a_{\alpha \beta}^{j} x^{\alpha} x^{\beta} \quad(j, \alpha, \beta=1,2)
$$

for which, from (2) and (3), we have that $V=0$. Therefore, by virtue of conditions $\mu \neq 0, V=0$ and in accordance with (4) the conditions (5) 
imply the following relations among the comitants $\mu, H, G$ and $F$ :

(7) $G^{2}-6 F H=0,3 H^{2}-2 \mu G=0,2 G^{3}+27 \mu F^{2}-18 F G H=0$.

We intend to show that for system (6) conditions (7) imply

$$
H=F=G=0 .
$$

Indeed, multiplying the first relation of ( 7$)$ by $-2 G$ and summing it with the third relation we obtain

$$
F(9 \mu F-2 G H)=0 .
$$

If $F=0$, then from (7) it follows that $H=G=0$. Otherwise, taking into account (7) we have

$4 H\left(G^{2}-6 F H\right)+9 F\left(3 H^{2}-2 \mu G\right)+2 G\left(2 G^{3}+27 \mu F^{2}-18 F G H\right)=3 F H^{2}=0$.

Therefore, we have obtained that $H=0$ and from (7) it again follows that $F=0$. Thus, conditions (8) are valid for system (6) if its singular point $(0,0)$ is of the fourth multiplicity and vice versa.

Let us denote by $\lambda_{1}$ and $\lambda_{2}$ the eigenvalues corresponding to the singular point $(0,0)$ of system $(6)$.

Case I. $\lambda_{1}^{2}+\lambda_{2}^{2} \neq 0$. According to [1] by applying a linear transformation and rescaling, system (6) can be written as follows

$$
\begin{aligned}
& \frac{d x}{d t}=g x^{2}+2 h x y+k y^{2}, \\
& \frac{d y}{d t}=y+l x^{2}+2 m x y+n y^{2},
\end{aligned}
$$

for which we have $F=0, G=g\left(g x^{2}+2 h x y+k y^{2}\right)$. By condition (8) we receive $g=0$ and calculating the values of $H$ and $\mu$ we obtain

$$
H=h l(2 h x+k y)=0, \mu=l\left(4 h^{2} m-4 h k n+k^{2} l\right) \neq 0 .
$$

Thus, we have $h=0, k l \neq 0$ and by scaling of the parameters, system (10) can be put in the form

$$
\begin{aligned}
& \frac{d x}{d t}=y^{2}, \\
& \frac{d y}{d t}=y+x^{2}+2 m x y+n y^{2},
\end{aligned}
$$

for which the conditions for distinguishing the topological classes through the parameters $m$ and $n$ are found in [9]. Namely, it occurs in the following: 
Proposition 3 [9]. A phase portrait of quadratic system (11) in the Poincare disk is up to a homeomorphism given by:

Figure 1 if $\bar{\eta}>0, X<0, Y>0$;

Figure 2 if $\bar{\eta}>0, X<0, Y \geq 0$ or $\bar{\eta}>0, X \geq 0$;

Figure 3 if $\bar{\eta}=0, R \neq 0, X<0, Y<0, Z \geq 0$;

Figure 4 if $\bar{\eta}=0, R \neq 0, X<0, Y<0, Z<0$;

Figure 5 if $\bar{\eta}=0, R \neq 0, X<0, Y>0$ or $\bar{\eta}=0, R \neq 0, X \geq 0$;

Figure 6 if $\bar{\eta}<0$, or $\bar{\eta}=R=0, \bar{L} \neq 0$,

where

$$
\begin{aligned}
\bar{\eta} & =-4 m^{3}+4 m^{2} n^{2}-36 m n+32 n^{3}-27, \\
R & =\left(3 m-4 n^{2}\right) x^{2}-(2 m n+9) x y-\left(m^{2}+6 n\right) y^{2}, \\
\bar{L} & =-x^{3}+2 n x^{2} y-m x y^{2}+y^{3}, X=2 n^{3} \\
Y & =m n, Z=\left(32 n^{3}+4 m^{2} n^{2}+27\right) n^{2}\left(m^{2}+6 n\right) .
\end{aligned}
$$

It is easy to prove the following assertion:

Proposition 4. The following statements hold.

1) The consequences of conditions

a) $n<0$, $m n<0$; b) $n \geq 0$ or $n<0$, $m n \geq 0$

are equivalent to the consequences of conditions

a) $m>0, m n<0$; b) $m n \geq 0$ or $m n<0, m<0$, respectively.

2) If $\bar{\eta}=0$ then the consequences of conditions

a) $n<0, m n<0,\left(32 n^{3}+4 m^{2} n^{2}+27\right)\left(m^{2}+6 n\right) \geq 0$;
b) $n<0, m n<0,\left(32 n^{3}+4 m^{2} n^{2}+27\right)\left(m^{2}+6 n\right)<0$; are equivalent to the consequences of conditions

a) $m>0, m n<0,\left(2 m^{3}+18 m n+27\right) m\left(m^{2}+6 n\right) \geq 0$;

b) $m>0, m n<0,\left(2 m^{3}+18 m n+27\right) m\left(m^{2}+6 n\right)<0$, respectively.

Indeed, the truth of the first statement of Proposition 4 follows directly from the expressions of the corresponding conditions. To prove the second one we assume that $\bar{\eta}=0$. According to (12) we have $-4 m^{3}+4 m^{2} n^{2}-36 m n+32 n^{3}-27=0$. Therefore, we have that $4 m^{2} n^{2}+32 n^{3}+27=2\left(2 m^{3}+18 m n+27\right)$ which proves Proposition 4. 
For system (11) the following comitants can be calculated:

$$
\begin{aligned}
\eta & =-4 m^{3}+4 m^{2} n^{2}-36 m n+32 n^{3}-27, \\
M & =\left(3 m-4 n^{2}\right) x^{2}-(2 m n+9) x y-\left(m^{2}+6 n\right) y^{2}, \\
L & =-x^{3}+2 n x^{2} y-m x y^{2}+y^{3}, D_{1}=\frac{7}{3} m n, \\
D_{2} & =\frac{2}{27} m^{3}, D_{3}=\frac{14}{243}\left(2 m^{3}+18 m n+27\right) m\left(m^{2}+6 n\right) .
\end{aligned}
$$

Taking into account Proposition 4 and relations (12) and (13), we deduce that the conditions of Theorem for the realization of each of the phase portraits of system (11) are equivalent to the corresponding conditions of Proposition 3. Thus, the Theorem is valid for system (1) with one non-zero eigenvalue of the singular point of multiplicity 4 .

Case II. $\lambda_{1}=\lambda_{2}=0$. According to [1] by applying a linear transformation and rescaling (6) we have

$$
\begin{aligned}
& \frac{d x}{d t}=y+g x^{2}+2 h x y+k y^{2}, \\
& \frac{d y}{d t}=l x^{2}+2 m x y+n y^{2},
\end{aligned}
$$

for which have $F=0, G=l\left(l x^{2}+2 m x y+n y^{2}\right)$. By virtue of conditions (8) we have $l=0$ and by calculating the values of $H$ and $\mu$ we obtain

$$
H=g m(2 m x+n y)=0, \mu=g\left(g n^{2}-4 h m n+4 k m^{2}\right) \neq 0 .
$$

Thus, we have $m=0, g n \neq 0$ and by scaling of the parameters, system (14) becomes

$$
\begin{aligned}
& \frac{d x}{d t}=y+g x^{2}+2 h x y+k y^{2}, \\
& \frac{d y}{d t}=y^{2},
\end{aligned}
$$

after the following substitution, $x=x_{1}-h y_{1}, y=g y_{1}, t_{1}=g t$ we have

$$
\begin{aligned}
& \frac{d x_{1}}{d t_{1}}=y_{1}+x_{1}^{2}+k y_{1}^{2}, \\
& \frac{d y_{1}}{d t_{1}}=y_{1}^{2} .
\end{aligned}
$$


As it was shown in $[\mathbf{9}]$ the phase portrait of system (15) corresponds to Figure 2 if $k<\frac{1}{4}$, to Figure 5 if $k=\frac{1}{4}$ and to Figure 6 if $k>\frac{1}{4}$. As for system (15)

$$
\eta=1-4 k, M=-x_{1}^{2}+x_{1} y_{1}+(3 k-1) y_{1}^{2}, D_{1}=0,
$$

we conclude that the phase portrait of system (14) corresponds to Figure 2 if $\eta>0$, to Figure 5 if $\eta=0$ and to Figure 6 if $\eta<0$. Taking into account the relation $D_{1}=0$ which holds for system (14), we conclude that the Theorem is valid in this case too. The Theorem is proved.

\section{References}

1. A. A. Andronov, E. A. Leontovich, I. I. Gordon And A. L. MAIER, "Qualitative theory of second-order dynamical systems," John Wiley \& Sons, New York, 1973.

2. V. A. Baltag And N. I. Vulpe, Affine-invariant conditions for determining the number and multiplicity of singular points of quadratic differential systems, Buletinul A.S. a R.M. ser. Matematica 1(11) (1993), 39-48.

3. D. Boularas, Iu. Calin, L. Timochouk and N. I. Vulpe, The polynomial basis of $T$-comitants for quadratic differential system, Conference of Applied and Industrial Mathematics, Romania-Moldova (1995), p. 28.

4. B. Coll, A. Gasull and J. Llibre, Quadratic systems with a unique rest point, Publ. Mat. UAB 32 (1988), 199-259.

5. G. Gurevich, "Foundation of the Theory of Algebraic Invariants," Noordhoff, Groningen, 1964.

6. P. DE JAGER, Phase portraits for quadratic systems with a high order singularity with two zero eigenvalues, J. Differential Equations 87 (1990), 169-204.

7. J. W. REYN, Phase portraits of quadratic systems with finite multiplicity one, Nonlinear Anal. (to appear).

8. K. S. Sibirsky, "Introduction to the Algebraic Theory of Invariants of Differential Equations," Manchester University Press, Manchester, 1988. 
9. N. I. Vulpe and I. V. Nikolaev, The topological classification of quadratic systems with a singular point of 4th multiplicity, Differential Equations 29(10) (1993), 1669-1674.

Mark Voldman:

Whitehead College

University of Redlands

1200 East Colton ave., Redlands

CA 92373 U.S.A.
Iu. T. Calin:

Institute of Mathematics

Academy of Sciences of Moldova

5 Academiei str., Chişinău

MD-2028 MOLDOVA

N. I. Vulpe:

Institute of Mathematics

Academy of Sciences of Moldova

5 Academiei str., Chişinău

MD-2028 MOLDOVA

Primera versió rebuda el 26 de Gener de 1996, darrera versió rebuda el 29 de Març de 1996 